\title{
Quand le sexe devient politique... Enjeux et périls du contre-rapport des femmes autochtones de Colombie aux Nations unies
}

Cuando el sexo se vuelve política... Desafíos y peligros del informe sombra de las mujeres indígenas de Colombia en la Naciones Unidas

When sex becomes political... Stake and peril of the indigenous women's shadow report in United Nations

\section{Anna Schmit}

\section{OpenEdition}

Journals

Edición electrónica

URL: http://journals.openedition.org/bifea/9609

DOI: $10.4000 /$ bifea.9609

ISSN: 2076-5827

Editor

Institut Français d'Études Andines

Edición impresa

Fecha de publicación: 1 agosto 2018

Paginación: 99-115

ISSN: 0303-7495

Referencia electrónica

Anna Schmit, "Quand le sexe devient politique... Enjeux et périls du contre-rapport des femmes autochtones de Colombie aux Nations unies », Bulletin de l'Institut français d'études andines [En línea], 47 (2) | 2018, Publicado el 08 agosto 2018, consultado el 04 noviembre 2020. URL : http:// journals.openedition.org/bifea/9609; DOI : https://doi.org/10.4000/bifea.9609

\section{(c)}

Les contenus du Bulletin de l'Institut français d'études andines sont mis à disposition selon les termes de la licence Creative Commons Attribution - Pas d'Utilisation Commerciale - Pas de Modification 4.0 International. 


\title{
Quand le sexe devient politique... Enjeux et périls du contre-rapport des femmes autochtones de Colombie aux Nations unies
}

\author{
Anna Schmit*
}

\begin{abstract}
Résumé
La Colombie adopte, en 1991, une nouvelle Constitution inscrivant, d'une part le caractère multiculturel de la nation et d'autre part, l'égalité des femmes et des hommes. Ce faisant, elle tend à réifier des catégories sociales, ce qui risque de défavoriser substantiellement l'exercice du droit des citoyennes autochtones. Afin de remédier à ce hiatus, une association locale de défense des droits humains des autochtones propose, en 2012, de renforcer l'alliance entre les femmes autochtones du pays pour formuler leur premier contre-rapport (shadow report) devant le Comité pour l'élimination des discriminations envers les femmes (CoEDEF). Cette action encourage les femmes à élever leurs droits sexuels et reproductifs au rang de sujet d'intérêts communs, nécessitant une intervention gouvernementale. Cet article propose d'éclairer la construction de leur positionnement politique, affirmant l'indissociabilité de l'autodétermination des peuples et de la liberté des femmes à disposer d'elles-mêmes.
\end{abstract}

Mots-clés : sexualité, femmes autochtones, multiculturalisme, Colombie, Nations unies

\section{Cuando el sexo se vuelve política... Desafíos y peligros del informe sombra de las mujeres indígenas de Colombia en la Naciones Unidas}

\section{Resumen}

En 1991, Colombia adopta una nueva Constitución que inscribe por un lado, el carácter multicultural de la nación y por otro, la igualdad de género. Paradójicamente, se produce una fragmentación entre las categorías sociales que desfavorece el ejercicio, del derecho de las mujeres indígenas. Con el propósito de rectificar este hiato, una asociación local de defensa de derechos humanos de los pueblos indígenas propone en 2012, reforzar una alianza entre las mujeres indígenas del país para formular su primer informe sombra (shadow report) ante el Comité de las Naciones unidas para la eliminación

IHEAL. Chercheure associée LAIOS/IIAC, 28 rue Saint-Guillaume, 75007, París. Email: aschmita@ gmail.com 
de todas las formas de discriminación hacia las mujeres (CEDAW). En esta acción, aquellas mujeres trataron de presentar sus derechos sexuales y reproductivos como un sujeto de interés común, que necesita una intervención del Gobierno. Este artículo propone analizar la construcción de este nuevo posicionamiento político, el cual afirma la indivisibilidad de la libre determinación de los pueblos y la libertad de las mujeres a disponer de su cuerpo.

Palabras clave: sexualidad, mujeres indígenas, multiculturalismo, Colombia, Naciones unidas

\title{
When sex becomes political... Stake and peril of the indigenous women's shadow report in United Nations
}

\begin{abstract}
In 1991, Colombia adopted a new Constitution, which enshrined the multicultural character of the nation and the equality of women and men. This has tended to produce a boundary between social categories that has disadvantages for Indigenous females citizens trying to exercise their rights. To remedy this legislative gap, in 2012, a local association of indigenous women's human rights proposed to strengthen the alliance between the indigenous women of the country in order to formulate a preliminary "shadow report" to present to the United Nations Comittee for the Elimination of Discrimination Against Women (CoEDAW). During this collective action, indigenous women tried to raise their sexual and reproductive rights to the level of matters of common interest, the respect for which requires governmental intervention. This article seeks to study the construction of this political position, which affirms the indivisibility of the peoples's free determination and women's liberty to have control over their bodies.
\end{abstract}

Keywords: sexuality, Indigenous women, Multicuturalism, Colombia, United Nations

La Colombie a adopté en 1991 une nouvelle Constitution, y inscrivant le multiculturalisme de la nation et l'égalité entre les hommes et les femmes. Ce texte prévoit l'adaptation des politiques publiques en fonction des différentes catégories sociales bénéficiaires, dans le respect du principe $d^{\prime}$ « égalité dans la différence » (Laurent, 2011 : 147). La nouvelle Constitution a pour vocation de protéger l'ensemble des citoyen.ne.s colombien.ne.s. Cependant, son interprétation et les programmes gouvernementaux qui en résultent tendent implicitement à figer les catégories sociales relevant des «droits des femmes » et celles relevant des « droits des peuples autochtones ». Le manque de reconnaissance politique des femmes autochtones 1 a eu pour effet d'affaiblir leur protection législative et d'obérer leur possibilité d'exercer leurs droits sexuels et reproductifs. Ceux-ci concernent le consentement à une relation sexuelle, la liberté de décider du nombre d'enfants, qu'elles veulent avoir et du moyen de contraception adaptée.

Cet article a l'ambition de rendre compte d'une action collective, engagée par un groupe de femmes autochtones de Colombie, visant à modifier cet état de fait. II

1 «Peuples autochtones » renvoie aux expressions, espagnole pueblos indígenas et anglaise, indigenous people. Ces dernières se réfèrent à une catégorie politique et relationnelle, en partie forgée grâce aux mobilisations indiennes aux Nations unies (Bellier, 2009). 
s'agit de l'élaboration du contre-rapport² des femmes autochtones de Colombie présenté au Comité des Nations unies pour l'élimination des discriminations envers les femmes (CoEDEF). Le cas de ce pays a été examiné par le Comité en octobre 2013, à Genève. La production de ce document a abouti au projet audacieux, non prévu initialement, de bouleverser les conceptions autochtones de la sexualité féminine et d'en faire un domaine d'intervention des politiques publiques. Un problème de taille s'est alors posé. Comment dénoncer les violences ayant cours dans les communautés, d'une part, sans décrédibiliser les luttes indiennes pour l'autodétermination des peuples aux Nations unies qui se montrent plutôt solidaires de ces luttes ; et d'autre part, en évitant que l'État ne se saisisse de leurs allégations pour saper l'autonomie autochtone, déjà fragile?

En construisant une analogie symbolique entre corps et territoire, ces femmes ont montré que les violences sexuelles dont elles étaient victimes rendaient improbable l'exercice par les autochtones de leur souveraineté (Vallejo \& García-Torres, 2017). Cette action leur a permis de se positionner par rapport à l'État, en exigeant de lui un accès à la justice, et de faire de leurs droits sexuels et reproductifs un sujet d'intérêt commun, nécessitant une intervention de la part de leur gouvernement, en concertation avec elles. En quoi l'analyse de la production du contre-rapport peut-elle éclairer les mécanismes par lesquels les femmes autochtones ont situé, dans la sexualité, l'élément essentiel de leur capacité d'agir politique?

L'article examinera comment, par un «jeu d'échelles», le sujet politique «femmes autochtones » se constitue face à leurs différents interlocuteurs : les peuples autochtones, le mouvement féministe, l'État colombien et les expertes du CoEDEF. Il mobilisera des données récoltées lors d'un travail de terrain anthropologique entre 2011 et 2015, réalisé avec l'association locale la Fuerza de Mujeres Wayúu3, qui a appelé à une mobilisation nationale des femmes autochtones. Ce travail nous a permis de dégager une ethnographie précise de cette action, en observant, entre 2012 et 2013, l'intégralité des évènements qui ont abouti à la formulation du contre-rapport et à son examen à l'ONU. Ces femmes y ont dénoncé, de manière inédite dans I'histoire des Nations unies, la mutilation génitale féminine dans un pays sud-américain. Cette pratique très minoritaire, observée notamment chez le peuple Embera, consiste à retirer le capuchon du clitoris à une nouvellenée. Premièrement, nous reviendrons sur le processus par lequel les militantes de la Fuerza de Mujeres Wayúu s'approprient le corpus des droits des femmes et requalifient ainsi certaines pratiques culturelles comme une violence de genre, portant atteinte à leur sexualité. Cette rencontre les encourage à diffuser, au sein du mouvement autochtone colombien, la Convention pour l'élimination

2 Un contre-rapport est un rapport rédigé par des organisations de la société civile et présenté aux Comités onusiens chargés d'observer et d'évaluer les pratiques des États dans l'application des Conventions internationales. II vient contrebalancer les conclusions des rapports présentés par les États.

3 La Fuerza de Mujeres Wayúu est une association locale constituée en organisation à but non lucratif depuis 2010. Elle regroupe plusieurs communautés wayúu, dispersées sur la péninsule de La Guajira. Depuis 2014, elle entend renforcer les liens politiques avec les Wayúu du Venezuela. 
des discriminations envers les femmes (CEDEF). Dans un deuxième temps, nous montrerons comment la dénonciation qu'elles font des mutilations génitales leur permet de faire passer l'expérience de leur sexualité du domaine de l'intime à celui de l'intervention publique. Ce faisant, elles proposent une vision alternative à la définition du multiculturalisme qui prévalait jusqu'alors. Dans un troisième temps, nous analyserons la transmission de leurs revendications devant la commission onusienne et l'écoute qu'elles ont obtenue, ou non, de la part de l'État colombien. L'analyse mettra ainsi en relief les enjeux et les périls de la mobilisation inédite des femmes autochtones de Colombie devant le CoEDEF.

\section{DE LA VICTIMISATION AUTOCHTONE À LA SUBJECTIVATION FÉMININE}

\section{1. Le genre des victimes}

La Fuerza de Mujeres Wayúu est à l'origine d'une action collective inédite de militantes autochtones colombiennes, réunies autour du recours au Comité onusien destiné à améliorer le droit des femmes. Cette association naît en 2007 d'un mouvement de victimes qui, dans La Guajira4, dénonce les exactions commises par les groupes paramilitaires et exige de l'État une réparation collective répondant aux critères propres de l'« approche différentielle » autochtone wayúu. Elle incarne une initiative populaire de participation démocratique dans un contexte de conflit armé. Le conflit armé colombien résulte de l'accaparement foncier par les strates dominantes (composées des notabilités régionales, nationales et internationales) et de la répression armée avec laquelle leurs émissaires font face aux demandes de participation politique populaire. Différents acteurs armés structurent les dynamiques belliqueuses et entendent exercer un contrôle sur les populations et les territoires ruraux, y compris ceux reconnus comme appartenant aux peuples autochtones. On compte parmi ces acteurs : les guérillas, armées subversives ; les narcotrafiquants, susceptibles d'influencer les dynamiques belliqueuses en altérant les jeux d'alliances économiques et politiques ; I'armée nationale, subordonnée au pouvoir exécutif ; et enfin, les paramilitaires, supplétifs des forces de l'ordre. Ces derniers émergent dans les années 1980 comme une coalition formée par des propriétaires terriens, des militaires et des individus issus de milieux criminels (Grajales, 2016). Durant les mandats du président Uribe, la classe dirigeante, soucieuse d'échapper aux dénonciations publiques dont faisait l'objet sa proximité avec les paramilitaires, s'empressa d'entamer des négociations avec ces groupes, en vue de leur démobilisation, ce qui a abouti à la loi 975 de 2005. Une période d'ouverture de post-conflit a alors été déclarée et s'est concrétisée par la mise

4 La Guajira est une région frontalière avec le Venezuela, située au nord de la Colombie sur la côte des Caraïbes. Elle abrite le peuple autochtone Wayúu, lequel regroupe environ 250000 personnes. Dès la fin des années 1990, des groupes armés paramilitaires s'immiscent dans la vie des Wayúu pour s'approprier leur commerce et pour monopoliser le contrôle des pistes traversant le territoire autochtone, de façon à faciliter l'exportation de drogues par les ports naturels de la région. 
en place d'une justice transitionnelle, à laquelle seraient dorénavant soumis les programmes gouvernementaux (Lecombe, 2013). Sous la pression des organisations de défense des droits humains, la réparation due aux victimes fut intégrée au cadre juridique de ces accords. La loi définissait donc l'aménagement des peines pour les paramilitaires et la « réparation intégrale» pour les victimes (Lecombe, 2013). Malgré les nombreuses critiques qu'elle a soulevées, cette loi a eut le mérite d'ouvrir un espace politique à la myriade d'associations de victimes, nouvellement créées par la société civile.

La Fuerza de Mujeres Wayúu s'est d'abord constituée comme un collectif de victimes autochtones contre l'oppression paramilitaire dans La Guajira. Sa composition, majoritairement féminine, est liée à la manière dont le conflit colombien a déchiré le tissu social guajiro. Les communautés wayúu sont formées par des matrilignages gouvernant de manière autonome leur espace territorial ${ }^{5}$. Ceux-ci s'insèrent dans des systèmes d'alliance entre familles, activés en cas de conflit. Les alliés participent aux guerres interfamiliales et/ou contribuent aux processus de justice compensatrice (Guerra Curvelo, 2002 ; Perrin, 1980). La persistance des menaces paramilitaires a contraint de nombreux hommes à quitter temporairement la région alors que les femmes se sont employées à entamer des démarches auprès des institutions colombiennes pour réclamer des réparations. C'est dans la quête de reconnaissance du statut de victime de guerre, demandé par plusieurs familles wayúu, que le collectif de la Fuerza de Mujeres Wayúu a pris forme. Selon les militantes, la nouvelle donne, introduite par les bouleversements provoqués par la guerre, leur a ouvert la possibilité de chercher des alliances en dehors des canaux traditionnels et d'inscrire leur action dans les réseaux élargis de la coopération internationale.

Les membres du collectif ont construit un discours victimaire, s'appuyant sur leur appartenance autochtone. La surreprésentation de femmes a attiré l'attention d'organisations féministes internationales, qui ont manifesté leur désir d'inclure ce collectif dans leurs programmes de coopération. Dès 2007, le collectif a perçu des subventions, allouées par le Fonds Global pour les Femmes. En 2009, I'organisation féministe norvégienne FOKUS I'a inclus à son programme visant I'amélioration, dans un contexte de conflit armé, des capacités organisationnelles de diverses associations de femmes (déplacées, autochtones, artistes, ex-combattantes...). Ce programme avait pour objectif de former ces collectifs à la défense des droits des femmes et de générer une réflexion sur les modalités d'application de la normativité internationale en matière de participation des femmes dans la résolution des conflits ${ }^{6}$. En échange de financements réguliers, la Fuerza a dû se transformer, en 2010, en une organisation officielle de défense des droits humains spécialisée dans la défense des droits des femmes autochtones.

5 Le terme matrilignage désigne une filiation établie par la lignée de la mère (Fox, 1972) et il ne doit pas être confondu avec un pouvoir exercé par des femmes même si ce type de filiation peut leur conférer une « autorité morale» (Mathieu éd., 2007).

6 Entretiens menés avec les militantes de la Fuerza entre 2011 et 2013 et avec la coordinatrice du programme FOKUS, février 2012 et mars 2013. 


\section{2. Le moment de la subjectivation et l'inclusion du droit des femmes}

La transformation du mouvement social en ONG (Adjamagbo \& Calves, 2012) et I'intégration d'une perspective de genre ont conduit la Fuerza à croiser le corpus des droits collectifs reconnus aux peuples autochtones et celui des droits individuels reconnus aux femmes. Progressivement, un discours politique s'est formalisé autour d'une identité sociale intersectionnelle, formée par le croisement des catégories subordonnées «femmes » et « autochtones » (Crenshaw \& Bonis, 2005 ; Purtschert \& Meyer, 2009).

Le rapprochement entre le collectif et les organisations féministes a conduit les militantes de la Fuerza à interroger leur appartenance autochtone à travers le prisme de leur appartenance de genre, et à nommer certaines des violences dont elles étaient l'objet sous le terme générique de "violence faite aux femmes ». Les témoignages recueillis lors de l'enquête font état de la tenue, dès 2008, de réunions internes entre les militantes. Elles nomment ces moments de partage ronión pour désigner le temps de loisir succédant au temps de travail, la reunión (réunion) (Schmit, 2016 : 450-452). La ronión est un espace de liberté, identifié par Laure Bereni et Anne Revillard sous le terme de free space (2012 : 25). Elle est vécue comme un moment intime où les militantes apprennent à se connaître et à s'identifier les unes aux autres. Les roniones leur ont donné l'occasion de discuter des problématiques qu'elles affrontaient dans leur vie quotidienne. Elles y ont mis en exergue les mauvais traitements qui jalonnaient les liens amoureux, la brutalité qui marquait souvent leur relation avec leur conjoint et la sexualité qu'elles assimilaient à un domaine de potentielle souffrance. S'y mêlaient des abus sexuels incestueux, des rapports sexuels forcés et la menace concrète de viols que représentait, pour elles, la présence d’hommes armés sur leur territoire (Falquet, 2006).

En leur proposant un modèle de décryptage des situations personnelles par une lecture de genre, les alliances établies avec FOKUS ont stimulé le processus de subjectivation par lequel les militantes de la Fuerza se sont saisies des droits des femmes. La subjectivation renvoie à l'incorporation d'éléments, rendus subjectifs par un sujet individuel ou collectif. Elle permet à ce sujet de réinscrire dans un cadre général son histoire personnelle. Elle participe à la construction d'un récit collectif dans lequel des histoires individuelles prennent sens (Tassin, 2014 : 169). Les militantes autochtones colombiennes ont analysé les effets du conflit armé sur leur vie, non plus seulement à l'aune du racisme structurel persistant en Colombie à l'encontre des peuples autochtones mais en incorporant également une dimension de genre, articulant les violences externes perpétrées par les acteurs du conflit et celles internes, exercées au sein des communautés. C'est ce qu'on montré Sabine Masson (2009), Shanon Speed (2008) ainsi que Shanon Speed et al. (2006) sur d'autres terrains. 


\section{MOBILISATION AUTOCHTONE AUTOUR DE LA CEDEF}

Ce processus de subjectivation des militantes de la Fuerza les a menées à se saisir du droit international des femmes et à proposer au mouvement indien de produire un contre-rapport sur la situation des femmes autochtones en Colombie. Ce document a inscrit la sexualité comme un domaine d'intervention publique, en rendant explicite la continuité entre les violences vécues à l'intérieur des communautés et celles provoquées par le conflit armé. L'objectif était de dénoncer les violences intracommunautaires, sans que cette dénonciation ne porte atteinte à l'autonomie autochtone. Les militantes ont positionné leur plaidoyer dans le combat politique des peuples autochtones pour leur autodétermination (Vallejo Real \& García-Torres, 2017). En contestant la manifestation sexuelle de la domination masculine, elles ont questionné la manière dont le multiculturalisme était compris par les autorités de leur peuple et par les agents institutionnels, et ont cherché à insuffler de nouvelles manières de le comprendre et de le mettre en œuvre.

\section{1. Naissance d'une mobilisation}

En 2010, la fondatrice de la Fuerza a suivi une formation sur la portée et l'usage de la CEDEF, proposée par l'Institut d'éducation des droits des femmes (Women Human Rights Institut - WHRI), hébergé par I'Université de Toronto. L'Institut organise, chaque année, une formation coordonnée par une avocate spécialisée en droit international des femmes. La militante wayúu a été une des premières femmes autochtones à s'y inscrire. Elle a, par la suite, porté le projet d'organiser cette formation en Colombie afin que les femmes autochtones puissent connaître les droits des femmes et profité de l'agenda onusien, prévoyant l'examen du cas colombien par le CoEDEF en octobre 2013, pour concrétiser ce projet. Avec le concours du WHRI et l'appui de FOKUS, la Fuerza a coordonné en septembre 2012 la « première semaine CEDEF pour les femmes autochtones de Colombie ». Une dizaine de représentantes d'organisations autochtones, venues de différents lieux de Colombie, y ont assisté. De cette action a émergé le projet de formuler un contre-rapport qui serait transmis au CoEDEF. Cette action collective a contribué à nouer une alliance à long terme entre les femmes autochtones de l'ensemble du pays.

Ce projet a alors pris un caractère national, en impliquant des femmes d'autres régions, peuples et organisations. Dans l'optique de le mener à bien, ces femmes se sont donné plusieurs types de rendez-vous. Soit elles finançaient des réunions entre elles avec le concours de leur organisation autochtone et/ou de leur partenaire de coopération ${ }^{7}$; soit elles profitaient de l'agenda autochtone pour se retrouver, discuter de leur action et susciter une plus large adhésion

7 « $1^{\text {ère }}$ semaine CEDEF » Cabo de la Vela, dans La Guajira, du 17 au 22 septembre 2012, Réunion à Caldas 26-27 novembre 2012, Réunion d'élaboration du contre-rapport, La Mina 23-25 mai 2012. 
au projet ${ }^{8}$. Nos propos se basent sur les observations que nous avons faites lors de toutes ces rencontres qui leur ont permis de légitimer l'action en soi, d'en clarifier les objectifs, d'élargir son public, et surtout de débattre des thèmes que le document final devrait aborder (Schmit, 2015).

\section{2. Construction d'un objet d'intérêt commun}

Ces réunions ont donné lieu à la constitution d'espaces de liberté dans lesquels un partage d'expérience commune mettait en évidence les préoccupations qu'il fallait retranscrire dans le contre-rapport : la faible participation politique des femmes autochtones, la précarité de leur accès à la justice et les difficultés liées à leur sexualité. Les militantes ont identifié des pratiques culturelles exerçant une grande violence à leur encontre, comme les mutilations génitales pratiquées dans quelques communautés autochtones. Elles ont abordé ce problème pour contester l'ordre en vigueur dans leur société autochtone et renforcé par le conflit qui nie la légitimité de leurs désirs sexuels. Ces femmes ont souligné la régularité avec laquelle se passer de leur consentement à une relation sexuelle représente une norme sociale valide. Elles ont placé l'expérience d'une sexualité brimée au cœur de la dynamique relationnelle du pouvoir qui assujettit leur existence par un contrôle exacerbé sur leur corps (Tabet, 1998 ; Héritier, 2002 ; 2002 ; Mathieu, 2014). En demandant à l'État de faciliter la mise en place d'espaces de concertation dans lesquels ces thèmes pourraient être discutés et débattus avec les autorités autochtones, les militantes ont construit un lien indissociable entre sexualité, affirmation de soi et politique. En effet, elles ont estimé que les violences contre les femmes à l'intérieur de leur communauté augmentent leur vulnérabilité face à des acteurs extérieurs et tendent à saper les luttes pour I'autonomie autochtone. Elles ont dénoncé les viols commis par ces hommes, l'augmentation de la prostitution qu'ils entraînent ainsi que les grossesses forcées et la monoparentalité contrainte qui en découlent.

Cependant, le Sommet idéologique des peuples (février 2013) a marqué une rupture dans les efforts pour constituer la violence intracommunautaire comme un objet d'intérêt commun nécessitant une intervention étatique. Il a été difficile d'obtenir l'adhésion des organisations nationales autour de la dénonciation de ce qui pouvait se passer dans des communautés déjà fortement marginalisées et subalternisées en Colombie. L'inscription dans un projet globalement féministe d'une action réalisée à partir du mouvement autochtone a suscité quelques inquiétudes chez les leaders d'organisations nationales. En effet, leurs homologues masculins se montrent souvent réticents à l'idée d'inclure les revendications des femmes à l'agenda politique autochtone. Par crainte d'être accusées de phagocyter les luttes autochtones, ces dernières ont préféré limiter le contre-rapport à la dénonciation des violences perpétrées par les acteurs armés (Schmit, 2016).

8 VIII Congrès de I'ONIC octobre 2012, Rencontre des femmes autochtones La Mina 17 novembre 2012, le Sommet des peuples autochtones février 2013, Espace de Concertation 10 avril 2013. 
Au cours de la rencontre de mai 2013, nous avons pu observer une série de positions sur ce sujet, reflétant la variation des places occupées par les organisations et les militantes dans l'espace autochtone national. Une militante du peuple Zenu a présenté les conclusions du travail effectué avec les autres femmes de son organisation :

La violence psychologique a pour conséquece que les femmes se sentent mises à l'écart et isolées dans leur propre maison. Leur liberté d'expression est bafouée. Dans les couples, une maltraitance envers les femmes réduit à néant leur estime de soi.

La violence psychique s'exerce avec des mots. Ces violences ont fait beaucoup de mal et ont soumis les femmes à une profonde solitude et au mépris de leur belle-famille.

La violence physique est celle qui se réalise par le biais de gestes et de désignation. Elle fait obstacle à la libre circulation des femmes. Les menaces se transforment fréquemment en agressions physiques.

Il y a violence sexuelle lorsque les femmes qui sont en couple sont physiquement fatiguées, qu'elles ne veulent pas avoir de relation sexuelle et que leur conjoint les y oblige.

La majorité des femmes n'osent pas dénoncer ces violences, de peur que ces mauvais traitements ne deviennent plus fréquents (Militante Zenu, mai 2013).

Le discours de cette femme a provoqué l'émoi de l'assemblée mais a placé les organisatrices de l'événement dans l'embarras. Une d'entre elles a répondu :

Ceci est la problématique que nous rencontrons dans nos communautés, elle représente la manière dont les femmes se sentent discriminées, humiliées. Il est très important que nous y pensions, mais nous ne pouvons pas présenter cette facette dans notre rapport parce que le gouvernement peut s'en prévaloir pour saper l'autonomie des autochtones (Militante Fuerza mai 2013).

Cette réponse de la part d'une organisatrice exprime bien le manque de consensus sur la question. À partir du Sommet des peuples, I'organisation nationale a exprimé son désaccord à inscrire la violence intracommunautaire dans le contre-rapport, alléguant qu'une telle dénonciation risquait de porter atteinte au mouvement autochtone. Ces divergences ont eu des répercussions sur les opinions des militantes de la Fuerza. Pour certaines, dénoncer les violences intracommunautaires contre les femmes risquait de nuire aux luttes autochtones, pour d'autres, inclure ces violences était, au contraire, un moyen de les renforcer. Finalement, le contre-rapport a évoqué la violence intracommunautaire et plus spécifiquement, les pratiques qui :

[...] en viennent à léser nos vies, comme les mutilations génitales, la pelona (rite durant lequel les cheveux d'une adolescente entrant dans la puberté sont arrachés), la violence, la maltraitance, les mariages forcés avec des enfants, 
l'inceste, les limitations et les interdictions de participation politique (Contrerapport «Derechos humanos, discriminación y violencia», 2013 : 17).

Le problème pointé est que lorsque la justice coutumière ne prend pas en compte ces violences, les femmes ont tendance à recourir à la justice ordinaire, ce qui tend à affaiblir la crédibilité des autochtones devant leur État (Sierra, 2004). Selon elles, I'inclusion de ces violences par les instances judicaires autochtones devient un moyen de renforcer les structures de gouvernance autochtone et la légitimité politique des autochtones face à leur État.

\section{3. Plaidoyer et participation démocratique}

Les extraits d'entretiens de terrain rapportés ci-dessus éclairent la prise de position qu'adoptent les femmes autochtones pour tenter de dépasser leur condition de marginalité et s'ériger en sujet politique avec qui l'État devra dorénavant composer (Ciavollela, 2010). À travers la rédaction d'un plaidoyer international (Keck \& Sikkink, 1998), les femmes autochtones de Colombie ont produit une analyse intersectionnelle des discriminations dont elles étaient la cible, articulant la subalternité de leur position dans la société dominante avec celle marquant leur condition au sein de leur peuple (Speed, 2008 ; Vallejo Real \& García-Torres, 2017). Pour elles, cette action a manifesté une exigence de justice sociale et démocratique, favorisant leur reconnaissance comme des actrices à part entière, participant à toutes les « interactions de la vie sociale»(Fraser, 2012). Cette dernière dimension invite à reconnaître la formation d'un contre-public (Fraser, 2011) à la croisée du féminisme et du mouvement autochtone, ce qui incite à revoir les catégorisations juridiques. Les militantes remettent en cause une vision du multiculturalisme selon laquelle les discriminations et les violences exercées à l'encontre des femmes autochtones sont une affaire privée, devant se régler uniquement dans les enceintes judiciaires des peuples, sans que l'État n'ait à intervenir. Au contraire, elles expriment la conviction que la formation d'une société plus juste, régulée par un État de droit fonctionnant sur des bases démocratiques, ne peut se réaliser sans I'accession de leurs problèmes au rang d'affaires publiques et sans leur concours actif, en collaboration avec l'État, pour les résoudre. Il a été demandé à l'État, non pas de se substituer aux autorités autochtones pour garantir aux femmes l'accès à la justice, mais de les accompagner, en intervenant dans les communautés, de manière concertée avec elles:

Notre discours doit être plus cohérent, nous devons délimiter les interventions de l'État. En tant qu'autochtones, nous pouvons prendre nos propres mesures; ce que nous voulons est que l'État garantisse que ces mesures se prennent. Par exemple, si nous disons : « cette année tant de fillettes ont été violées par leur grand-père », comment l'État peut-il aider les communautés à régler ce type de problèmes et à garantir que ces choses ne se reproduisent plus? (Militante, novembre 2012).

L'enjeu pour les femmes autochtones a été la constitution de leur sexualité en un domaine d'intérêt public pour lequel une intervention du gouvernement était 
souhaitable. Leur objectif était de concourir à cette intervention tout en évitant les risques potentiels que pouvait représenter, pour l'autonomie autochtone, une ingérence de l'État.

\section{LUTTES ET TENSIONS AUX NATIONS UNIES}

Les observations 9 menées au Palais des Nations ont montré la difficulté des femmes autochtones à porter leurs revendications, et ont fait apparaître de manière saillante, le refus de l'État de les considérer comme actrices politiques. Trois temps successifs ont marqué l'enquête en terrain onusien. Le premier fut celui d'une réunion informelle entre les quatre femmes autochtones présentes à Genève, deux militantes de la Fuerza et deux militantes de l'organisation nationale. Le second temps fut celui qualifié dans le jargon onusien de « déjeuner informel », c'est-àdire une présession, réunissant les représentantes des organisations de la société civile et les expertes du Comité, en vue de préparer l'examen. Le troisième temps fut celui du dialogue entre l'État colombien et les expertes, en session plénière.

\section{1. Politiser la sexualité aux Nations unies}

Avant que ne débute la présession, la délégation autochtone s'était réunie dans un des bureaux mis à disposition pour l'événement afin de décider des thèmes qui mériteraient d'être discutés lors de l'examen. Contrairement aux désaccords survenus lors du Sommet idéologique des peuples, les quatre représentantes du mouvement autochtone se sont entendues pour aborder la question de leur sexualité. Elles ont considéré le contrôle sexuel auquel elles sont souvent soumises comme le principal vecteur des discriminations dont elles sont la cible. La possibilité, pour chaque personne, de se concevoir et de se penser indépendamment du rapport qui la lie à l'autre sexe pose les bases psychiques de l'élévation de soi en sujet politique (Gramsci, 2014 [1948-1951] : 2147-2150). Dans ce cadre, la question des droits sexuels et reproductifs et de l'accès à ces droits prend toute son importance. La maîtrise par les femmes autochtones de leur sexualité devient la voie ouvrant sur l'autonomie du sujet politique «femme autochtone » (Del Re, 2007 : 1-6). Les militantes ont estimé que la domestication de leur corps, qui consacre l'absence de plaisir comme une garantie du maintien des traditions, constitue une entrave à leur épanouissement personnel, prohibition exacerbée dans les communautés autochtones où les mutilations génitales sont pratiquées.

Traiter des mutilations génitales aux Nations unies pour en faire un objet d'attention prioritaire demande l'élaboration d'un vocabulaire stratégique, intelligible pour les expertes, afin d'éviter qu'une erreur d'interprétation ne sape la crédibilité des autochtones. Les femmes autochtones ont voulu éviter qu'elles ne deviennent un argument justifiant colonisation et domination (Mathieu, 2014 : 125-131). II a

9 Je remercie le soutien du SOGIP lors de ma mission de terrain aux Nations unies. 
donc été indispensable d'adopter une perspective pédagogique pour expliquer au Comité l'importance de débattre des schémas culturels au sein des peuples et insister auprès des expertes sur les raisons données par les sages-femmes pratiquant l'excision. Ces femmes, convaincues du bienfondé des soins ainsi prodigués à la nouvelle-née, assimilent le clitoris à un organe qui entre en concurrence avec le pénis des hommes et de ce fait, porteur de déséquilibre mental pour les femmes (Mathieu, 2014 : 133-150). L'intervention des autochtones devant les expertes devait donc aboutir au développement de programmes gouvernementaux d'éducation sexuelle dans les communautés autochtones et à la constitution d'espaces de dialogue favorisant une remise en question des mutilations dans les communautés, sans stigmatiser les sages-femmes autochtones dans la société nationale.

\section{2. Des sexes mutilés à la parole ébréchée}

Après cette réunion interne, la délégation autochtone s'est jointe à l'ensemble des organisations civiles colombiennes dans la salle prévue pour le déjeuner informel. Plusieurs associations de victimes, de féministes et des ONG de défense des droits humains étaient présentes. Les débats ont été rapidement axés sur la sexualité des femmes. À plusieurs reprises, les femmes autochtones se sont efforcées de signifier qu'elles avaient quelque chose à dire sur des questions de portée générale, posées par les expertes. Cependant, elles n'ont pu prendre la parole qu'à la fin de la rencontre, pour répondre à une question qui leur était directement adressée par une experte ; cette question portait sur leur accès à la santé et sur la production par le gouvernement colombien de données chiffrées illustrant la situation. Une militante de la Fuerza y répondit en profitant de ce temps de parole pour souligner l'absence d'une justice adéquate pour les femmes autochtones.

Durant les dernières minutes de ce déjeuner, les autochtones ont introduit la question des mutilations génitales dans le débat et ont formulé une demande claire au Comité :

Nous voulons donner des informations concernant les mutilations qui se produisent dans certaines communautés autochtones en Colombie. Nous considérons que le Comité a la compétence de travailler avec nous pour les faire cesser. II ne s'agit pas de considérer les praticiennes comme des criminelles. C'est à cause de leur manque de connaissance, qu'elles pratiquent l'excision du clitoris. Nous avons besoin d'appui et nous voudrions qu'une recommandation soit adressée aux institutions nationales afin qu'elles prennent en compte cet enjeu dans les programmes de politiques publiques (octobre 2013).

Les expertes ont alors demandé davantage de précisions sur la manière dont on procédait à ces mutilations. La gravité des faits exposés, au moment où le temps du déjeuner s'achevait, a donné lieu à une réunion spontanée entre la délégation autochtone et l'experte rapporteuse de la session colombienne. Celle- 
ci a proposé d'utiliser comme levier d'action l'article 5 de la Convention, relatif à l'élimination des discriminations insufflées par des pratiques coutumières, et d'émettre une sanction à l'encontre de l'État colombien s'il ne prenait pas des mesures concrètes pour éradiquer cette pratique. Le Comité ne pouvait pas émettre de réelles sanctions. Néanmoins, il pouvait proposer aux autres organismes onusiens de soumettre les aides de la coopération internationale au respect d'une recommandation. Les femmes autochtones ont refusé cette option qui, selon elles, produirait des effets néfastes. Elles ont demandé à l'experte de faciliter la mise en place d'espaces de dialogue entre les communautés pratiquant les mutilations génitales et l'État.

La description du déjeuner montre deux aspects de l'intervention des autochtones. Le premier renvoie aux difficultés des femmes autochtones à inscrire leurs propres revendications dans l'espace du déjeuner. Ces difficultés ont fait resurgir les discriminations structurelles qui les touchent et leur position marginalisée dans le mouvement féministe colombien. Le second aspect illustre la ténacité avec laquelle les femmes autochtones, en s'efforçant d'inscrire la lutte contre les mutilations génitales dans les priorités du Comité pour en faire un élément des programmes publics, ont réussi à imposer ce thème dans les préoccupations communes (Fraser, 2011 ; 2012). Elles ont inscrit l'intégrité et l'autonomie corporelle des femmes dans un « rapport de pouvoir » et l'on attendait du Comité qu'il parvienne à en modifier les termes (Roman, dir., 2014). Elles ont réaffirmé la mission de l'État de garantir l'intégrité physique et morale des ressortissantes autochtones, mise à mal par les pratiques de mutilations génitales.

\section{3. Sexualité et femmes autochtones dans le dialogue constructif}

L'examen, par le Comité, de l'action de l'État colombien devait se faire par un « dialogue constructif» (Delzangues \& Möschel, 2014 : 59), entre expertes et représentants de l'État. Le Comité interrogea l'État sur sa bonne observance des articles 1 à 16 de la CEDEF, en prenant en compte le contexte général du pays et les avancées significatives, évaluées à l'aune du précédent rapport. La délégation colombienne était composée de dix femmes, travaillant dans des ministères et des institutions nationales. Elle était présidée par la directrice du Haut conseil présidentiel pour l'équité des femmes (ACPEM).

La question des mutilations génitales féminines, évoquée pour la première fois par le Comité dans le cas d'un pays d'Amérique latine, fut soulevée à la lecture de l'article 5. L'experte a demandé des comptes à l'État colombien, en insistant sur la complexité de ce thème. La directrice de I'ACPEM a souligné le travail entamé par l'ICBF (l'entité institutionnelle chargée de veiller à la protection des enfants) depuis 2007, dans les régions où se pratiquent les mutilations. Selon elle, les institutions colombiennes n'en prennent connaissance que lorsque la situation se révèle dangereuse pour l'enfant et que son transfert vers un centre de soin devient vital. Elle a précisé que, selon les principes constitutionnels, il revient aux autorités autochtones de sanctionner cette pratique. Ainsi, il est souligné que 
l'État, même s'il accepte de prendre en compte ce problème, n'est pas compétent pour gérer ce genre de situation. Toutefois, la directrice a nuancé son propos en admettant qu'un travail avait été entamé avec les autorités autochtones. Celles-ci, ayant reconnu le caractère nocif des mutilations, ont accepté de participer à des actions de sensibilisation. La fonctionnaire a rappelé le rôle des sages-femmes dans le maintien de cette tradition.

Les expertes ont demandé davantage de précision sur les stratégies du gouvernement pour éradiquer la mutilation génitale car ce sujet n'avait pas été abordé dans le rapport officiel. Elles ont sollicité des informations sur les moyens de contrôler et de suivre les progrès réalisés sur le terrain. Elles ont regretté le manque de données chiffrées, pourtant essentielles, selon elles, à la mise en œuvre et au suivi de toute politique. La fonctionnaire colombienne a rebondi sur la responsabilité des autochtones et sur les difficultés pour le gouvernement de produire des chiffres en la matière :

Je répète que c'est une préoccupation du gouvernement, nous savons que nous devons élaborer avec plus de précision un état des lieux et en faire le suivi. Cependant, pour le moment, il est très difficile de pouvoir offrir une information détaillée basée sur un registre parce que l'accès à ces communautés est très difficile. Elles ne sont ni proches d'une hôpital ni d'un centre de santé. De plus, [les mutilations] sont pratiquées en cachette par les sages-femmes, lors des accouchements dans les communautés.

Ainsi que l'avaient craint les femmes autochtones, le discours de la directrice de I'ACPEM a fait porter sur les sages-femmes autochtones la responsabilité des mutilations. Selon elles, ce type de discours conduit inexorablement à accentuer la marginalisation dont leur peuple fait objet au sein des espaces internationaux.

À la fin de la session, une experte a mis en exergue la maltraitance intrafamiliale, insistant sur les liens entre justice et violence, révélés par les nombreux rapports d'ONG et par les témoignages des victimes, qui faisaient état de la prolifération de la violence privée et de la totale impunité dont bénéficient ses auteurs. Elle a demandé des précisions sur les mesures de protection prévues spécifiquement pour les femmes autochtones exposées à des sévices. La représentante de la Justice a formulé une réponse brève et confuse, en expliquant que la compétence revenait à la justice ordinaire mais que, conformément à la constitution, il était préférable d'assigner ces cas à la justice autochtone, s'ils s'étaient produits dans sa juridiction. Ces propos ambigus ont laissé planer de sérieux doutes sur les réelles possibilités de recours à la justice des femmes autochtones.

Ces exemples illustrent la nature du dialogue s'établissant entre les femmes autochtones et les représentantes de l'État colombien sous la médiation du Comité. La délégation colombienne refusa ici de prendre acte des demandes d'un groupe de citoyennes, dont elle annula la parole, en excluant sa requête de son cadre de compétence. Les préoccupations exprimées par ces femmes furent ainsi reléguées dans le domaine privé des peuples autochtones. Cependant, I'intervention des autochtones auprès des expertes et leur présence à cette session ont rendu visibles leurs revendications dans le domaine « public » et inscrit leur parole dans un 
espace hégémonique (Spivak, 2009 : 98), dont on espère qu'il favorisera par la suite leur visibilité devant leur État.

L'analyse de l'élaboration du contre-rapport des femmes autochtones et l'examen de l'État colombien par le CoEDEF montre comment émerge, à partir de la catégorie sociale «femme autochtone », ce que Nancy Fraser appelle un « contre-public subalterne». La reconnaissance de la condition victimaire a permis de concrétiser des alliances avec le mouvement féministe, alliances à la genèse du processus de subjectivation par lequel les militantes autochtones se sont appropriées du droit des femmes et en ont fait un outil pertinent d'analyse de leurs conditions de vie. La subjectivation de ce droit les a amenées à appréhender leur sexualité comme l'expression matérielle de leur position subalterne à l'intersection de différents rapports de pouvoir. Elles en ont fait un domaine d'intervention publique visant à modifier les normes sociales induisant des comportements qui leur sont préjudiciables. Comme le suggère Masson (2009) et Vellejo Real \& García-Torres (2017), elles ont introduit le respect des droits des femmes dans les luttes pour l'autodétermination des peuples.

Par un « jeu d'échelle», les femmes autochtones ont cherché à inclure les droits individuels des femmes dans le corpus des droits collectifs des peuples autochtones et ont exigé une protection de la part de l'État, par le biais de l'arbitrage onusien. Leur fragile adhésion à la dénonciation des violences sexuelles à l'intérieur des communautés a laissé place, le jour de l'examen, à une solide convergence. Toutefois, I'analyse de l'examen onusien montre les limites de l'émergence de ce contre-public dont les revendications spécifiques peinent à être pleinement prises en compte par l'État colombien. II n'en reste pas moins que l'action collective a le mérite d'avoir encouragé ces femmes à formuler leurs problèmes, à les porter devant les institutions publiques, et à se faire reconnaître comme « sujet politique » capable de participer à l'élaboration des politiques publiques qui lui sont destinées.

\section{Références citées}

ADJAMAGBO A. \& CALVES, A.-E., 2012 - L'émancipation féminine sous contrainte. Autrepart, 2, $\mathbf{n}^{\mathbf{0}}$ 61: 3-21.

BELLIER, I., 2009 - Autochtones. Espaces temps.net

BERENI, L. \& REVILLARD, A., 2012 - Un mouvement social paradigmatique ? Ce que le mouvement des femmes fait à la sociologie des mouvements sociaux. Sociétés contemporaines, $\mathbf{1}\left(\mathbf{n}^{\circ} \mathbf{8 5}\right)$ : 17-41.

CIAVOLELLA, R., 2010 - Les Peuls et l'État en Mauritanie, Une anthropologie des marges, 432 p. ; Paris-Milan : Karthala.

CONTRE-RAPPORT FMW, ONIC, OPIAC, 2013 - Una mirada a los derechos humanos de las mujeres indigenas colombianas. Informe sombre. Examen comité Cedaw 2013, 23 pp.; Bogotá agosto de 2013. Informe elaborado por mujeres indígenas 
de Colombia de la:Consejería Mujer Familia y Generación -organización Nacional Indígena de Colombia -ONICCoordinación Mujer, Familia y Niñez -Organización de los Pueblos Indígenas de la Amazonía Colombiana -OPIACSutsuin Jiyeyu Wayuu Fuerza de Mujeres Wayuu.

CRENSHAW, K. W. \& BONIS, O., 2005 - Cartographies des marges : intersectionnalité, politique de l'identité et violences contre les femmes de couleur. Cahiers du genre, 2, no 39: 51-82.

DEL RE, A., 2007 - Avortement et contraception. In : Dictionnaire Critique du féminisme (F. Laborie, H. Le Doaré, D. Senotier \& H. Hirata, éds.) : 1-6 ; Paris : PUF.

DELZANGLES, B. \& MÖSCHEL, M., 2014 - Le Comité pour l'élimination des discriminations envers les femmes : trente ans d'activités en faveur des femmes. In : La Convention pour l'élimination des discriminations à l'égard des femmes (D. Roman, éd.) : 4980 ; Paris : Éditions A. Pedone.

FALQUET, J., 2006 - Hommes en armes et «femmes de service » : tendances néolibérales dans l'évolution de la division sexuelle et internationale du travail. Cahiers du Genre, 1, $\mathbf{n}^{\circ} \mathbf{4 0}: \mathbf{1 5}-37$

FOX, R., 1972 - Anthropologie de la parenté. Une analyse de la consanguinité et de I'alliance, 276 p. ; Paris : Gallimard, « Les Essais ».

FRASER, N., 2011 [2005] - Qu'est ce que la justice sociale ? Reconnaissance et redistribution, 178 p. ; Paris : La Découverte.

FRASER, N., 2012 - Le féminisme en mouvements. Des années 1960 à l'ère néolibérale, 332 p.; Paris : La Découverte, «Politique et société».

GRAJALES, J., 2016 - Gouverner dans la violence. Le paramilitarisme en Colombie, 324 p. ; Paris : Karthala.

GRAMSCI, A., 2014 [1948-1951] - Quaderni del Carcere, Vol. 3, 3438 p. ; Torino : Einaudi.

GUERRA CURVELO, W., 2002 - La disputa y la palabra: la ley en la Sociedad Wayuu, 327 p. ; Bogotá : Ministerio de la Cultura.

HÉRITIER, F., 2002 - Masculin/Féminin II, Dissoudre la hiérarchie, 433 p. ; Paris : Odile Jacob.

KECK, M. E. \& SIKKINK, K., 1998 - Activist beyond borders: advocacy Networks in International Politics, 240 p. ; Ithaca : Cornell University Press.

LAURENT, V., 2011 - Dans, contre, avec l'Etat : mouvement indien et politique(s) en Colombie, vingt ans après. In : Le multiculturalisme au concret, un modèle latinoaméricain ? (C. Gros \& D. Dumoulin-Kervan (coords.) : 147-158 ; Paris : Presse Sorbonne Nouvelle.

LECOMBE D., 2013 - « Nous sommes tous en faveur des victimes » : usages sociaux et politiques de la justice transitionnelle en Colombie (2002-2010), 473 p. ; Paris : IEP. Thèse de doctorat en Science politique.

MATHIEU, N.-C. (éd.), 2007 - Une maison sans fille est une maison morte. La personne et le genre en sociétés matrilinéaires et/ou uxorilocales, 503 p. ; Paris : Éditions de la Maison des Sciences de I'Homme.

MATHIEU, N.-C., 2014 - L'anatomie politique 2. Usage, déréliction et résilience des femmes, 386 p. ; Paris : La Dispute. Collection « Le genre du monde».

MASSON, S., 2009-Sexe, race et colonialité. Point de vue d'une épistémologie postcoloniale latino-américaine féministe. In : Sexe, race, classe, pour une épistémologie de la domination (E. Dorlin, éd.) : 183-200 ; Paris : PUF, «Actuel Marx».

PERRIN, M., 1980 - La raison du plus fort est souvent la meilleure... Justice et vengeance chez les Indiens Goajiros. In : La Vengeance. Études d'ethnologie, d'histoire et de philosophie (R. Verdier, coord.) : 163-191; Paris : Éditions Cujas. 
PURTSCHERT, P. \& MEYER, K., 2009 - Différences, pouvoir, capital. Réflexions critiques sur I'intersectionnalité. In : Sexe, race, classe, pour une épistémologie de la domination (E. Dorlin, éd.) : 127-146 ; Paris : PUF. Collection «Actuel Marx».

ROMAN, D. (dir.), 2014 - La Convention pour l'élimination des discriminations envers les femmes, 370 p. ; Paris : Pedone.

SCHMIT, A., 2015 - La Fuerza de Mujeres Wayúu et la défense des droits fondamentaux des femmes autochtones en Colombie. Nuevo mundo, mundos nuevos, Colloque, mis en ligne le 01 décembre 2015, URL : http://nuevomundo.revues.org/68544; DOI : 10.4000 /nuevomundo.68544

SCHMIT, A., 2016 - «Teníamos el sueño de cambiar el mundo». Expérience militante de femmes autochtones de La Guajira - Colombie. Paris : EHESS. Thèse de doctorat en anthropologie politique.

SIERRA, T., 2004 - Haciendo justicia: interlegalidad, derecho y género en regiones indígenas, 477 p. ; México D. F.: CIESAS Miguel Ángel Porrúa.

SPEED, S., 2008 - Rights in rebellion. Indigenous Struggle \& Human Rights in Chiapas, 244 p.; Standforf University Press.

SPEED, S., HERNÁNDEZ CASTILLO, A. \& STEPHEN, L. M., 2006 - Dissident women: gender and cultural politics in Chiapas, 320 p. ; Austin : University of Texas press.

SPIVAK, G., 2009 - Les subalternes peuvent-elles parler ?, 109 p. ; Paris : Éditions Amsterdam.

TABET, P., 1998 - La construction sociale de l'inégalité des sexes. Des outils et des corps, 206 p. ; Paris-Montréal : L’Harmattan, Bibliothèque du féminisme.

TASSIN, E., 2014 - Subjectivation versus sujet politique : réflexion à partir d'Arendt et de Rancière. Tumultes, $\mathbf{2} \mathbf{n}^{\circ} \mathbf{4 3}:$ 157-173.

VALLEJO REAL, I. \& GARCÍA-TORRES, M., 2017 - Mujeres indígenas y neo-extractivismo petrolero en la Amazonía centro del Ecuador: Reflexiones sobre ecologías y ontologías políticas en articulación. Revista Brújula, Volumen 11: 43 p.; Davis: University of California. 